\title{
INVESTIGATIONS ON OPTICAL AND RADIO REFERENCE
}

\section{FRAMES}

\author{
S. PULIAEV ${ }^{1}$ AND A.H. ANDREI \\ Observatório Nacional \\ Rio de Janeiro - RJ, Brazil
}

\begin{abstract}
Features of the systematic errors of the Carlsberg Automatic Meridian Circle (CAMC) reference frame are investigated using a secondary radio reference frame defined by a set of astrometric VLA, VLBI and submillimetric positions of radio stars, taken from the literature. The radio positions of 62 stars are compared against the corresponding CAMC positions. The systematic errors of the CAMC catalogue are found to be generally smaller than 0.05 . The radio positions were also compared with the positions obtained with the Bordeaux Automatic Meridian Circle (BORD). It was found a good coincidence between (CAMC-Radio) and (BORD-Radio) systematic differences.
\end{abstract}

\section{Introduction}

The radio positions of selected stars have been used to study the general tie between radio and optical reference frames (Johnston et al., 1985; Morrison et al. 1990). Radio stars were also employed to reach a preliminary investigation on the existence of systematic rotations between the HIPPARCOS frame and the VLBI extragalactic reference frame (Lestrade et al., 1995). The number of stars in these investigations is equal to or less than 23, and constitutes on a difficulty for zonal studies, as previously pointed out (de Vegt and Gehlich, 1982).

\section{Radio Stars}

The group of 62 astrometric radio positions of stars was compiled carefully from the literature, being 32 from the VLA operating in the continuum mode, 16 from the VLA line mode observations, 5 obtained by VLBI, 6 from IRAM interferometer and 3 from Hat Creek interferometer. We assu-

${ }^{1}$ On leave from Pulkovo Observatory, St. Petersburg, Russia

\section{M. Wytrzyszczak, J. H. Lieske and R. A. Feldman (eds.),}

Dynamics and Astrometry of Natural and Artificial Celestial Bodies, 449, 1997.

(C) 1997 Kluwer Academic Publishers. Printed in the Netherlands. 
med that the collected group of radio observations of stars materialized a secondary celestial reference frame, referred to an extragalactic radio reference system obtained by VLBI observations.

About one hundred stars have had their radio position already determined, many of them have been observed several times during the last two decades by means of the VLA or VLBI. We retrieved this set of 62 radio stars because they were observed both with the Carlsberg and Bordeaux meridian circles. We have taken an unique J2000 position for each star, that is, the one we found most reliable in astrometrical sense. The criteria were the smallest error of radio position and the best coincidence of the optical and radio emitting centers, what depends mainly on the frequency band of observation or on the spectrum line. Bowers et al. (1983), from their VLBI observations, point out that $\mathrm{H}_{2} \mathrm{O}$ and $\mathrm{OH}$ line positions "occur at respectively larger distances from the star", thus we gave preference to $\mathrm{SiO}$ line $(86 \mathrm{GHz})$ positions. "The general properties of the $\mathrm{SiO}$ masers and the VLBI maps of $\mathrm{SiO}$ emission indicate that $\mathrm{SiO}$ masers are closely related to the underlying star" (Baudry et al., 1995).

\section{Optical Reference Frame}

We took the series of the Carlsberg Meridian Catalogues Nrs. 1-4 (1990), 5 (1991), 6 (1992), 7 (1993) and 8 (1994) as the optical reference frame to be compared with the radio positions of radio stars. We assumed that all the CAMC catalogues were built in the same system, defined by a single system of reference (the FK5 catalogue), a single instrumental system and the same method of reduction of observations. Therefore a compiled CAMC catalogue was made, combining the above mentioned series. Details of the method used are presented in Andrei et al., (1995). The catalogue consists of 117697 stars and is used at the Observatório Nacional/Brazil as the reference system in the astrometrical CCD observations of Solar System and extragalactic objects (Assafin et al., 1996). The resulting catalogue exhibits precisions of nearly 0. " 08 , comparable to those of some radio observations by VLA. The positions of these radio stars as given by observations at the Bordeaux Automatic Meridian Circle were also compared with the radio positions.

\section{Results of Comparison and Conclusions}

The optical positions of the radio stars were transformed to the epochs of their radio observations using the proper motions from either CAMC or BORD. The equatorial coordinates differences CAMC minus Radio and BORD minus Radio are shown in Figure 1, against right ascension (a), (c) and declination (b), (d). In the figure the smooth, fitting lines were obtained as the averages for a five points running bin. 

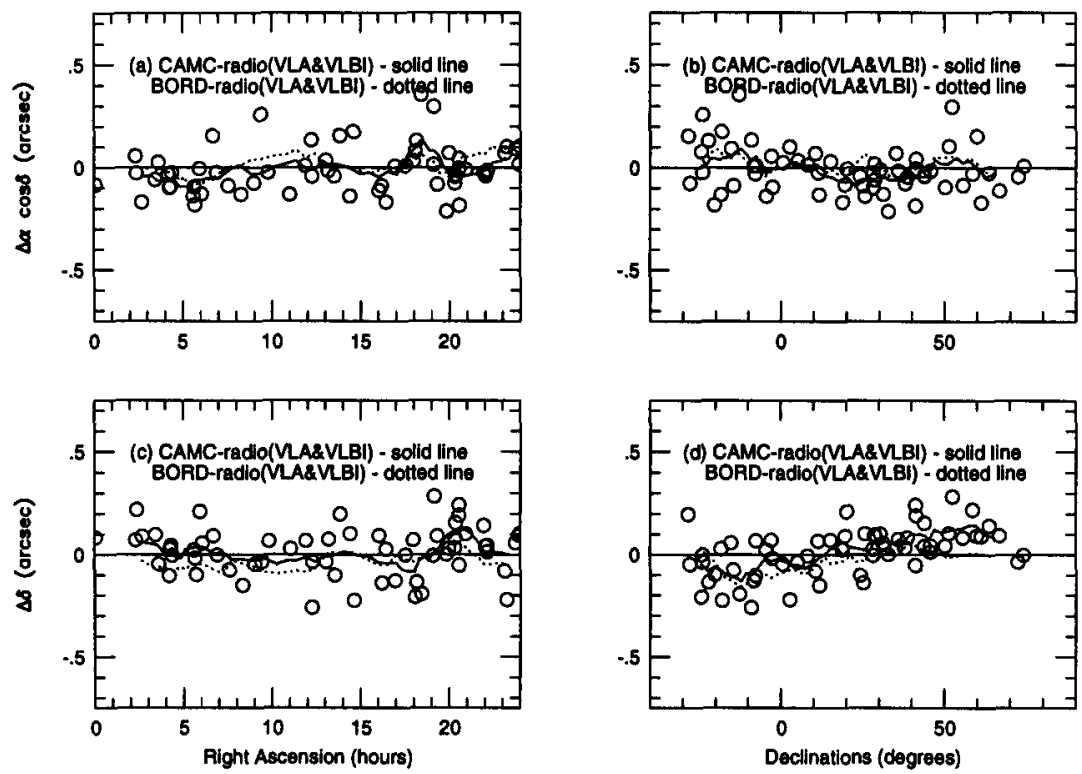

Figure 1. Optical minus Radio systematic differences. (CAMC-Radio) are presented by solid lines whereas (BORD-Radio) by dotted lines. The open circles are mark the (CAMC-Radio) individual positional differences.

The systematic deviations of the CAMC catalogue relatively to the radio positions are found to be generally less than 0 ". 05 . The analysis shows agreement between the radio results from the different facilities, accounting for the different error thresholds.

It was found a remarkable coincidence between the global systematic differences (CAMC-Radio) and the FK5 minus Hipparcos H30 preliminary version, (FK5-H30), (Lindegren et al., 1995), specially in $\Delta \delta_{\delta}$. The H30 space catalogue is of undisputed accuracy in comparison with ground based catalogues, so the (FK5-H30) represent the systematic errors of the FK5. Accepting that the (CAMC-Radio) truly represent the CAMC systematic errors, it is concluded that the later is well coincident with the FK5. Thus, it is found that the CAMC is aligned with the FK5 to an accuracy of about 0 " 05 , i.e. better than accuracy of the FK5. Finally, we point that the (CAMC-Radio) differences of the type $\Delta \alpha_{\delta}$ and $\Delta \delta_{\delta}$ as obtained here are close to recent results of similar investigations, as kindly supplied to the authors by Morrison (Argyle et al., 1996).

All the (BORD-Radio) systematic differences $\Delta \alpha_{\alpha}, \Delta \alpha_{\delta}, \Delta \delta_{\alpha}$ and $\Delta \delta_{\delta}$ are in good agreement with those from the (CAMC-Radio) comparison. This indicates that both the Carlsberg and Bordeaux observations are well linked with the FK5. In its turn, the FK5 has some small - up to 0.1 systematic errors, that were revealed by its comparison with the $\mathrm{H} 30$ and, 
likewise, in this work by the comparison of the CAMC and BORD (shown well aligned with the FK5) catalogue positions with the radio positions.

On the account of the quality and internal consistency of the collected radio positions of stars, it is verified that they made possible to determine the small systematic errors of the CAMC and BORD catalogues. In this sense, they constitute a secondary radio reference frame, formed mainly by astrometric VLA and VLBI and submillimetric determinations of positions of radio stars.

Finally, we calculated the set of three independent global rigid rotations around orthogonal axis, following Arias et al. (1988), in the sense CAMC and BORD onto Radio. Taking a right-handed triad of axis tied to the equatorial system, the rotations are given by the angles $\omega_{x}$, about the axis passing by the equinox; $\omega_{z}$, about a polar axis; and $\omega_{y}$, about the axis $(\alpha$ $=6 \mathrm{~h}, \delta=0^{\circ}$ ). The optical minus radio rotational parameters given by the (CAMC-Radio) differences are (in mas):

$$
\omega_{x}=+1 \pm 16, \quad \omega_{y}=+53 \pm 18 \quad \text { and } \quad \omega_{z}=+15 \pm 16
$$

Analogously, for the (BORD-Radio) differences, are obtained (in mas):

$$
\omega_{x}=+27 \pm 18, \quad \omega_{y}=+8 \pm 20 \quad \text { and } \quad \omega_{z}=-13 \pm 18
$$

Comparing with the corresponding set of values, independently obtained by Stone (1994), some evidence is revealed for a significant $\omega_{y}$ term for the FK5 based systems as compared to the extragalactic reference system.

Acknowledgements S. Puliaev thanks the CNPq/Brazil for the financial support under the contract 300017/93-6(NV).

\section{References}

Andrei A.H., Jilinski E.G., and Puliaev S.P.: 1995, Astron. J. 109, 428-433.

Assafin M., Vieira-Martins R., and Andrei A.H.: 1996, this volume.

Baudry A., Lucas R., and Guilloteau S.: 1995, Astron. Astrophys. 293, 594-604.

Bowers P.F., Johnston K.J., and Spencer J.H.: 1983, Astrophys. J. 274, 733.

Carlsberg Meridian Catalogues No 1-4, 5, 6, 7, 8 : 1990, 1991, 1992, 1993, 1994, Copenhagen University Observatory, Royal Greenwich Observatory and Real Instituto y Observatório de la Armada en San Fernando.

de Vegt C. and Gehlich U.K.: 1982, Astron. Astrophys. 113, 213.

Johnston K.J., de Vegt C., Florkowski D.R., and Wade C.M.: 1985, Astron. J. 90, 2381.

Lestrade J.-F., Jones D.L., Preston R.A., Phillips R.B., Titus M.A., Kovalevsky J., Lindegren L., Hering R., Froeshlé M., Falin J.-L., Mignard F., Jacobs C.S., Sovers O.J., Eubanks M., and Gabuzda D.: 1995, Astron. Astrophys. 304, 182-188.

Lindegren L., Roser S., Schrijver H., Lattanzi M.G., van Leeuwen F., Perryman M.A.C., Bernacca P.L., Falin J.L., Froeshle M., Kovalevsky J., Lenhardt H., and Mignard F.: 1995, Astron. Astrophys. 304, 44-51.

Morrison L.V., Argyle R.W., Requieme Y., and Mazurier J.M.: 1990, Astron. Astrophys. 236, 256-260.

Requieme Y. and Mazurier J.M.: 1991, Astron. Astrophys. 89, 311-318.

Stone R.C.: 1994, Astron. J. 108, 313-325. 